\title{
25. Wandel von Teilhabe und Integration älterer Menschen - ein politikorientiertes Fazit
}

\author{
Frank Berner, Katharina Mahne, Julia K. Wolff \& Clemens Tesch-Römer
}

Im vorliegenden Buch werden empirische Analysen und Befunde in einer großen thematischen Bandbreite präsentiert: Es geht um Einkommen und Vermögen, um Erwerbsarbeit und den Übergang in den Ruhestand, um Beziehungen innerhalb der Familie, um soziale Netzwerke, um freiwilliges Engagement und Freizeitaktivitäten, um die Wohnsituation und die nachbarschaftlichen Beziehungen, um die Übernahme von Sorgeaufgaben (Unterstützung, Pflege, Enkelkinderbetreuung), um Lebensqualität und Wohlbefinden, um verschiedene Aspekte der gesundheitlichen Entwicklung, um Ansichten über andere Generationen sowie um Altersbilder. Das große Themenspektrum dieses Buches spiegelt den Facettenreichtum der Lebenssituation älterer Menschen wider. Darüber hinaus macht die thematische Breite deutlich, dass Senioren- oder Alternspolitik eine Vielzahl von Handlungsfeldern berührt. In diesem abschließenden Kapitel werden verschiedene konkrete Zielsetzungen und Handlungsfelder der Alternspolitik genauer beschrieben und mit den empirischen Befunden der voranstehenden Kapitel verknüpft.

Es ist ein zentrales übergreifendes Ziel der Alternspolitik, die Teilhabe und Integration älterer Menschen in allen Lebensbereichen $\mathrm{zu}$ fördern und zu sichern. Das vorliegende Buch schafft dafür die wissenschaftlichen Grundlagen. Der empirische Reichtum dieses Buches beschreibt zum einen, auf welche Weise und in welchem Maße Teilhabe und Integration von Menschen in der zweiten Lebenshälfte realisiert werden und welche Folgen dies hat. Zum anderen werden dabei die vielfältigen Voraussetzungen für Teilhabe und Integration beschrieben. Wer dieses Buch liest, kann also Antworten finden sowohl auf die Frage „Was wurde erreicht?“ als auch auf die Frage „Was ist noch zu tun?“.

Im Folgenden stellen wir zunächst Ziele und Entwicklungen in vier zentralen Handlungsfeldern der Alternspolitik dar, in denen es darum geht, die Teilhabe und Integration von Menschen in der zweiten Lebenshälfte zu unterstützen. Im zweiten Abschnitt dieses Kapitels diskutieren wir die Ergebnisse des Deutschen Alterssurveys 2014 mit Blick auf diese Handlungsfelder. Daraus leiten wir Empfehlungen zur Verbesserung und zum Erhalt von Teilhabe und Integration in der zweiten Lebenshälfte ab. Weiterhin nehmen wir zwei zentrale Determinanten sozialer Ungleichheit in den Blick: Lebensalter und Bildungsstand. Abschließend werden daraus einige Schlussfolgerungen gezogen.

\subsection{Handlungsfelder und politische Ziele der Alternspolitik}

Die Förderung und Sicherung von Teilhabe und Integration in der zweiten Lebenshälfte sind übergreifende Ziele einer Politik für ältere Menschen. Viele politische Maßnahmen in verschiedenen Handlungsfeldern sind an diesen Zielen ausgerichtet. Wie in Kapitel 1 erläutert wird, be- ziehen sich die Auswertungen in diesem Buch schwerpunktmäßig auf vier Handlungsfelder:

a) Verlängerung des Erwerbslebens,

b) Vereinbarkeit von Beruf und Sorgetätigkeiten, 
c) Gleichstellung von Frauen und Männern sowie

d) Selbstständiges Wohnen im Alter.

Im Folgenden werden zunächst zentrale Herausforderungen und politische Ziele in diesen vier Handlungsfeldern dargestellt.

\subsubsection{Verlängerung des Erwerbslebens}

Der Zeitpunkt und die verschiedenen Wege des Übergangs vom Erwerbsleben in den Ruhestand sind ein Dauerthema im Schnittbereich zwischen Arbeitsmarkt-, Alterssicherungs- und Alternspolitik. Im Jahr 2007 wurde das Gesetz zur Anpassung der Regelaltersgrenze an die demografische Entwicklung und zur Stärkung der Finanzierungsgrundlagen der gesetzlichen Rentenversicherung beschlossen, auf dessen Grundlage nun langsam und langfristig die Regelaltersgrenze in der gesetzlichen Rentenversicherung angehoben wird.

In den politischen Debatten über die Anhebung der Regelaltersgrenze in der gesetzlichen Rentenversicherung ging es im Wesentlichen um die Frage: Ist es vertretbar, die Regelaltersgrenze anzuheben? Derzeit scheint die Debatte differenzierter zu werden - inzwischen geht es nicht mehr nur um die Frage, ob es eine Verlängerung des Erwerbslebens geben sollte oder nicht, als vielmehr darum, für den Übergang vom Erwerbsleben in den Ruhestand mehr Optionen bereitzustellen. Die abschlagfreie Rente ab 63 für langjährig Versicherte, die im Jahr 2014 eingeführt wurde und häufig als eine Abkehr vom Paradigma einer längeren Lebensarbeitszeit interpretiert wird, kann vielleicht eher als Teil einer Differenzierung von Möglichkeiten für den Ausstieg aus dem Erwerbsleben angesehen werden (Bundesministerium für Arbeit und Soziales 2014).

Bislang wurden bestehende Möglichkeiten für einen flexiblen Austritt aus dem Erwerbsleben wenig genutzt (Czepek \& Weber 2015). Um hier Abhilfe zu schaffen, hat sich die Regierungskoalition im November 2015 darauf verständigt, die Übergänge in den Ruhestand zu flexibilisieren (und die sogenannte ,Flexi-Rente einzuführen). Dabei soll es Arbeitnehmerinnen und Arbeitnehmern erleichtert werden, eine Teilrente zu beziehen und zugleich in Teilzeit arbeiten können. Mit der Flexi-Rente soll weniger der Zeitpunkt eines abrupten Übergangs vom vollen Erwerbsleben zum vollen Ruhestand flexibler gemacht werden, vielmehr soll dieser Übergang gestreckt und gleitender gemacht werden.

\subsubsection{Vereinbarkeit von Beruf und Sorgetätigkeiten}

Die meisten älteren Menschen, die auf Unterstützung und Pflege angewiesen sind, bekommen diese Unterstützung und Pflege von ihren Angehörigen. Etwa 71 Prozent aller Bezieherinnen und Bezieher von Leistungen aus der sozialen Pflegeversicherung wurden im Jahr 2013 teilweise oder ausschließlich von Angehörigen gepflegt (Statistisches Bundesamt 2013). Damit ist die Familie nach wie vor der , größte Pflegedienst' (Wetzstein, Rommel, \& Lange 2015). Die informelle Hilfe und Unterstützung innerhalb von Familien ist dabei noch nicht einmal berücksichtigt.

Allerdings ist die Angehörigenpflege aus verschiedenen Gründen immer weniger selbstverständlich. In der Pflegepolitik setzt sich vor diesem Hintergrund derzeit die Ansicht durch, dass zur Bewältigung des zukünftigen Pflegebedarfs nicht auf die, rein familiale Pflege' gesetzt werden kann. Vielfach wird inzwischen empfohlen, systematisch sogenannte gemischte Pflegearrangements zu fördern (Rothgang, Müller, \& Unger 2012). In gemischten Pflegearrangements wird die Pflege und Unterstützung alter Menschen von einem Netzwerk aus Angehörigen, professionellen Pflegekräften, Nachbarn und Nachbarinnen sowie freiwillig Engagierten geleistet. Indem die Pflege und Unterstützung alter Menschen auf mehrere Schultern verteilt wird, sollen pflegende Angehörige entlastet werden, ohne dass pflege- und unterstützungsbedürftige Menschen ihr gewohntes Umfeld verlassen und in eine stationäre Einrichtung ziehen müssen. 
Darüber hinaus müssen weitere Anstrengungen unternommen werden, die Vereinbarkeit von Berufstätigkeit und Sorgearbeit zu erleichtern. In diesem Bereich hat sich in den vergangenen Jahren Einiges getan: Mit dem Familienpflegezeitgesetz wurde im Jahr 2012 ein neues Modell der befristeten Freistellung vom Arbeitsplatz eingeführt. Die bestehenden Regelungen wurden mit dem Gesetz zur besseren Vereinbarkeit von Familie, Pflege und Beruf, das seit dem 1. Januar 2015 in Kraft ist, weiter entwickelt und besser verzahnt.

\subsubsection{Gleichstellung von Männern und Frauen}

Die Bundesregierung hat sich zum Ziel gesetzt, die Gleichstellung von Männern und Frauen in allen gesellschaftlichen Bereichen zu verwirklichen. Dies soll vor allem durch mehr Partnerschaftlichkeit zwischen Männern und Frauen in Familie und Beruf erreicht werden. Dazu wurden in den vergangenen Jahren richtungsweisende und $\mathrm{zu}$ gleich zum Teil kontrovers diskutierte Maßnahmen ergriffen. So wurde etwa mit der Einführung des Elterngeldes zum Jahr 2007 erreicht, dass mehr Männer als früher nach der Geburt eines Kindes Elternzeit nehmen. Seit dem 1. Mai 2015 ist das Gesetz für die gleichberechtigte Teilhabe von Frauen und Männern in Führungspositionen in der Privatwirtschaft und dem öffentlichen Dienst in Kraft - damit sollen mehr Frauen als bislang in Führungspositionen tätig sein. Beim Girls‘ Day und beim Boys' Day sollen Jugendlichen beiderlei Geschlechts solche Berufe näher gebracht werden, die gerade nicht als typisch für das jeweilige Geschlecht gelten. Solche Maßnahmen entfalten ihre Wirkungen vor allem langfristig und zunächst eher bei jüngeren Kohorten. Dennoch ist es daneben unerlässlich, geschlechtsspezifische Unterschiede in den Lebenssituationen älterer Männer und Frauen zu beschreiben und ihre Entwicklung zu beobachten.

Im Mai 2015 hat die Bundesregierung die Sachverständigenkommission zur Erstellung des Zweiten Gleichstellungsberichts der Bundesregierung eingesetzt. Diese Kommission hat auch den Auftrag, die Umsetzung der Empfeh- lungen aus dem Ersten Gleichstellungsbericht der Bundesregierung zu evaluieren. Dabei werden insbesondere Übergänge im Lebensverlauf in den Blick genommen (etwa die berufliche Orientierung, der Einstieg in das Berufsleben, die Phase der Familiengründung, der Ausstieg aus dem Erwerbsleben, die Übernahme von Pflegeaufgaben). Es ist deshalb zu erwarten, dass die Sachverständigenkommission in ihrem Bericht Vorschläge für weitere politische Maßnahmen entwickeln wird, um die Gleichstellung von Männern und Frauen auch in der zweiten Lebenshälfte und im Alter voranzubringen.

\subsubsection{Selbstständiges Wohnen im Alter}

In verschiedenen Umfragen zeigt sich in leichten Variationen immer wieder dasselbe Bild: Die meisten Menschen wünschen sich, im Alter so lange es geht in ,der eigenen Wohnung' zu leben. Damit ist nicht unbedingt Wohneigentum im engeren Sinne gemeint, sondern vielmehr die private Häuslichkeit, sei es zur Miete oder im selbstgenutzten Wohneigentum. Es ist dementsprechend ein zentrales Ziel der Alternspolitik, die Voraussetzungen dafür zu schaffen, dass ältere Menschen selbstbestimmt und möglichst lange selbstständig leben und wohnen können. Dieses Ziel kann nur durch ein Zusammenwirken von Entwicklungen und Maßnahmen in verschiedenen Bereichen erreicht werden: Erstens müssen bezahlbare Wohnungen vorhanden sein. Dabei muss berücksichtigt werden, dass viele ältere Menschen nur mit geringen finanziellen Ressourcen (Einkommen und Vermögen) ausgestattet sind. Zweitens sollten die Wohnungen oder Häuser die entsprechenden baulichen Voraussetzungen haben. Dies bedeutet, sie sollten möglichst barrierereduziert gestaltet sein, dies betrifft vor allem die Ausstattung und die Bewegungsflächen in den Sanitärräumen sowie die Erreichbarkeit der Wohnung und der verschiedenen Räume innerhalb der Wohnung möglichst ohne Treppen. Drittens ist es hilfreich, wenn in der direkten Umgebung der Wohnung eine gute Versorgung mit Gütern (z. B. Lebensmitteln) und Dienstleistungen (z. B. Arztpraxen, 
Apotheken, Beratungsstellen) möglich ist. Viertens sollte die Wohnumgebung so gestaltet sein, dass der soziale Austausch innerhalb der Nachbarschaft erleichtert wird.

\subsection{Ergebnisse des Deutschen Alterssurveys (DEAS) 2014}

Die Befunde des DEAS 2014 können mit den oben genannten politischen Handlungsfeldern in Beziehung gesetzt werden. Die Interpretation der Ergebnisse ist dabei immer angeleitet von dem übergreifenden Ziel der Alternspolitik, Teilhabe und Integration in der zweiten Lebenshälfte zu fördern und zu sichern.

\subsubsection{Verlängerung des Erwerbslebens: Befunde des DEAS 2014}

Länger $\mathrm{zu}$ arbeiten ist durch gesetzgeberische Maßnahmen seit den 1990er Jahren sowie die begleitenden öffentlichen Debatten zu einer gesellschaftlichen Norm geworden. Inzwischen scheinen mehr und mehr Menschen diese Norm $\mathrm{zu}$ verinnerlichen. Immer mehr Erwerbstätige planen ihren Renteneintritt mit 65 Jahren oder später: 1996 planten 18,2 Prozent der befragten Erwerbstätigen, mit 65 Jahren oder später aus dem Erwerbsleben auszuscheiden - im Jahr 2014 waren es schon 39,5 Prozent. Mit 66 Jahren oder später aus dem Erwerbsleben auszusteigen planten 19,0 Prozent der Erwerbstätigen (vgl. Kapitel 4). Im gleichen Zeitraum hat sich sowohl in der Altersgruppe der 54- bis 59-Jährigen wie auch in der Altersgruppe der 60- bis 65-Jährigen der Anteil der Erwerbstätigen deutlich erhöht: jeweils um etwa 20 Prozentpunkte (vgl. Kapitel 3).

Zudem sind seit einiger Zeit immer mehr Menschen im Ruhestand erwerbstätig, also zeitgleich mit dem Bezug einer Altersrente (Engstler \& Romeu Gordo 2014; Scherger \& Hokema 2014), was sich auch in den DEAS-Daten zeigt. Der Anteil der Personen, die im Ruhestand erwerbstätig sind, ist von 5,1 Prozent im Jahr 1996 auf 11,6 Prozent im Jahr 2014 deutlich angestiegen (vgl. Kapitel 3). Insgesamt hat sich die Beteiligung älterer Menschen am Erwerbsleben deutlich erhöht. Diese Entwicklung ist zu einem großen Teil auf die arbeitsmarktpolitischen und alterssicherungspolitischen Reformen seit der Jahrtausendwende zurückzuführen, die auf eine stärkere Erwerbsbeteiligung älterer Arbeitnehmerinnen und Arbeitnehmer sowie auf eine Verlängerung des Erwerbslebens zielten.

Allerdings gibt der DEAS 2014 auch Hinweise darauf, dass die Rahmenbedingungen für einen längeren Verbleib im Erwerbsleben noch nicht in jeder Hinsicht optimal sind. So ist von den im Ruhestand Erwerbstätigen ein überdurchschnittlich großer Teil selbstständig tätig (2014: 41,5 Prozent). Lediglich 26,4 Prozent der im Jahr 2014 im Ruhestand Erwerbstätigen arbeiten bei dem Arbeitgeber, bei dem sie auch schon vor dem Ruhestand tätig waren. Hier besteht Handlungsbedarf, denn Menschen, die auch nach dem Renteneintritt weiter arbeiten wollen, wollen dies am liebsten bei ihrem bisherigen Arbeitgeber tun (Bäcker 2015).

Es gibt daneben auch Anzeichen dafür, dass ein längerer Verbleib im Erwerbsleben nicht für alle Erwerbstätigen möglich oder sinnvoll ist - oder nicht von allen Erwerbstätigen gewollt wird: Der Anteil derjenigen, die einen Ausstieg aus dem Erwerbsleben mit weniger als 65 Jahren planen, hat sich zwar zwischen 1996 und 2008 stark verringert (von 63,7 Prozent auf 42,4 Prozent), ist zwischen 2008 und 2014 aber stabil geblieben (vgl. Kapitel 4). Die weitere Entwicklung wird zeigen, ob diese Gruppe langfristig doch immer kleiner wird oder ob sich hier ein ,Sockel' von Erwerbstätigen bildet, die auch in Zukunft planen, mit weniger als 65 Jahren aus dem Erwerbsleben auszusteigen. Es wäre sicherlich aufschlussreich, mehr über die Motive dieser Menschen zu erfahren. Zudem ist zwischen 1996 und 2014 der Anteil der Erwerbstätigen, die unmittelbar vor dem Rentenbezug in der Arbeitslosigkeit sind, von 3,6 Prozent auf 15,5 
Prozent stark angestiegen. Diese Entwicklung betrifft vor allem Frauen in den ostdeutschen Bundesländern: Bei dieser Gruppe hat der Renteneintritt aus der Arbeitslosigkeit von 1,0 im Jahr 1996 auf 39,7 Prozent im Jahr 2014 zugenommen (vgl. Kapitel 4).

In der öffentlichen Debatte über die Anhebung der Regelaltersgrenze stand in den vergangenen Jahren vor allem die Frage im Vordergrund, unter welchen gesundheitlichen Voraussetzungen Arbeitnehmerinnen und Arbeitnehmer bis zum 67. Lebensjahr arbeiten können. Vor diesem Hintergrund ist beachtenswert, dass sich gerade in den mittleren $\mathrm{Al}$ tersgruppen (bei den 54- bis 59-Jährigen und den 60- bis 65-Jährigen) bei mehreren Gesundheitsdimensionen (Anzahl von Erkrankungen, funktionale Gesundheit und depressive Symptome) von $2008 \mathrm{zu} 2014$ eine Verschlechterung abzeichnet (vgl. Kapitel 8 und 11). Auch wenn sich die Gesundheit von jüngeren Kohorten gegenüber älteren Kohorten im Vergleich zu 1996 weiterhin verbessert hat und sich bei den über 65-Jährigen zudem auch von $2008 \mathrm{zu} 2014$ positive Entwicklungen zeigen, sollte man die Veränderung der Gesundheit der erwerbsfähigen Bevölkerung weiter beobachten und negativen Trends entgegenwirken. Verbesserungen der gesundheitlichen Prävention und der Rehabilitation, die mit der geplanten Flexi-Rente (siehe oben) eingeführt werden sollen, sind vor diesem Hintergrund sinnvoll.

Die hier aufgegriffenen Erkenntnisse, beruhend auf den Daten des DEAS 2014, lassen den Schluss zu, dass verschiedene Gruppen von Erwerbstätigen unterschiedliche Bedürfnisse beim Übergang vom Erwerbsleben in den Ruhestand haben. Die entscheidende Frage ist weniger, ob die Erwerbstätigen länger arbeiten können oder es nicht können. Es wird vielmehr immer offensichtlicher, dass manche Menschen es können, andere jedoch nicht, dass manche Menschen es gerne wollen und andere es müssen. Es wäre sicherlich sinnvoll, sich nicht auf die angehobene Regelaltersgrenze der gesetzlichen Rentenversicherung als zentralen normierten Zeitpunkt für den Übergang vom Erwerbsleben in den Ruhestand zu konzentrieren, sondern verschiedene gleichberechtigte Zeitpunkte für den Übergang vom Erwerbsleben in den Ruhestand zu institutionalisieren.

\subsubsection{Vereinbarkeit von Beruf und Sorgetätigkeiten: Befunde des DEAS 2014}

Die Pflege und Unterstützung innerhalb der $\mathrm{Fa}$ milie hat in Deutschland einen hohen Stellenwert. Dem entsprechend wird ein großer Teil der auf Pflege angewiesenen Menschen von ihren Angehörigen gepflegt. Die Daten des DEAS 2014 zeigen jedoch, dass mit dem sozialen Wandel die Angehörigenpflege in verschiedener Hinsicht weniger selbstverständlich wird.

Zum einen werden die Wohnentfernungen zwischen erwachsenen Kindern und ihren Eltern von $1996 \mathrm{zu} 2014$ kontinuierlich größer (vgl. Kapitel 14). Dies lässt vermuten, dass für immer mehr Familien die Angehörigenpflege schwieriger $\mathrm{zu}$ organisieren ist. Zweitens sinkt der Anteil derjenigen Menschen im mittleren Erwachsenenalter, die Kinder haben: Hatten im Jahr 1996 noch 87,2 Prozent aller 42- bis 47-Jährigen Kinder, waren es im Jahr 2014 nur noch 79,6 Prozent (vgl. Kapitel 14). Wer keine Kinder hat, kann im Bedarfsfall auch nicht von den eigenen Kindern gepflegt oder unterstützt werden. Für nachfolgende Kohorten ist also anzunehmen, dass sie bei Pflegebedarf im Alter weniger auf Töchter und Söhne als Unterstützerinnen beziehungsweise Unterstützer bauen können. Drittens sind immer mehr Menschen in der zweiten Lebenshälfte erwerbstätig, und zwar sowohl vor als auch nach dem Eintritt in den Ruhestand (vgl. Kapitel 3). Dies bindet bei diesen Menschen Zeitressourcen. Der Anstieg der Erwerbsquote vor dem Ruhestand ist insbesondere bei Frauen stark ausgeprägt, die auch im Jahr 2014 zu einem größeren Anteil als Männer Pflegeaufgaben übernehmen (vgl. Kapitel 12).

Zwischen 1996 und 2014 ist der Anteil der Personen, die mindestens eine andere gesundheitlich eingeschränkte Person unterstützen, von 12,3 Prozent auf 16,2 Prozent angestiegen (vgl. Kapitel 12). Eine stärkere Einbindung von Personen aus der weiteren Verwandtschaft, der Nachbarschaft oder dem Freundeskreis in die Unterstützung und Pfle- 
ge alter Menschen ist jedoch nicht erkennbar. Der Anteil derjenigen, die eine oder mehrere nichtverwandte Personen unterstützen oder pflegen, ist von 1996 bis 2014 weitgehend stabil geblieben: Etwa ein Fünftel der Menschen in der zweiten Lebenshälfte leisten diese Form der Unterstützung. Zugleich bleibt die Angehörigenpflege weiter eine zentrale Säule in der Pflege. Die Stellung der engsten Angehörigen bei der Pflege ist zwischen 1996 und 2014 eher größer als kleiner geworden. Eine Entwicklung hin $\mathrm{zu}$ gemischten Pflegearrangements kann derzeit aus den Daten des DEAS 2014 also nicht herausgelesen werden. Es ist deshalb umso wichtiger, zum einen die Rahmenbedingungen für gemischte Pflegearrangements zu verbessern und zum anderen eine breite gesellschaftliche Debatte über den Nutzen gemischter Pflegearrangements in Gang zu setzen. Im Siebten Altenbericht der Bundesregierung wird die Notwendigkeit gemischter Pflegearrangements thematisiert (Berner \& Hagen 2015) - der Siebte Altenbericht könnte genutzt werden, um eine entsprechende öffentliche Debatte voranzubringen.

Auch der Anteil derjenigen Menschen, die eine Berufstätigkeit mit Pflege oder Unterstützung verbinden, ist zwischen 1996 und 2014 angestiegen, und zwar von 7,0 auf 11,8 Prozent (vgl. Kapitel 12). Diese Entwicklung ist wohl darauf zurückzuführen, dass zum einen die Erwerbsbeteiligung insbesondere von Frauen und zum anderen die Zahl und der Anteil der auf Pflege angewiesenen alten Menschen angestiegen sind. Wenn eine Person berufstätig ist und zugleich Verantwortung bei der Pflege oder Unterstützung eines alten Menschen übernimmt, dann ist dies in der Regel mit einer nicht geringen Belastung dieser Person verbunden. Es ist deshalb begrüßenswert, dass die Bundesregierung in den letzten Jahren zur Verbesserung der Vereinbarkeit von Beruf und Pflege gesetzgeberisch aktiv war. Die Wirkungen der neu eingeführten Regelungen können allerdings erst in einigen Jahren verlässlich beurteilt werden.

Die praktische Sorge um Andere kann sich bei Menschen in der zweiten Lebenshälfte sowohl in der Unterstützung oder Pflege älterer Menschen zeigen als auch in der Betreuung von Kindern. Bei der Betreuung von Enkelkindern durch die Großeltern verläuft die Entwicklung von 1996 bis 2014 uneinheitlich: Während zwischen 1996 und 2008 der Anteil der Großeltern, die Enkelkinder betreuen, von 33,7 Prozent auf 24,7 Prozent zurückging, übernehmen im Jahr 2014 wieder mehr Großeltern die Betreuung von Enkelkindern (ein knappes Drittel, vgl. Kapitel 15). Da das durchschnittliche Übergangsalter zur Großelternschaft im Jahr 2014 bei etwa 53 Jahren liegt und außerdem die Erwerbstätigkeit im späteren Erwerbsalter zunimmt (vgl. Kapitel 3 und 4) fällt für einen wachsenden Teil von Großeltern der Betreuungsbedarf der Enkelkinder in eine Lebensphase, in der sie selbst noch einer Erwerbstätigkeit nachgehen. Im Vergleich der Jahre 1996 und 2014 zeigt sich, dass der Anteil von Großeltern bis 65 Jahre, der eine Betreuung von Enkelkindern mit Erwerbstätigkeit kombiniert, von 15,0 Prozent im Jahr 1996 auf 23,4 Prozent im Jahr 2014 angestiegen ist. Dabei sind jüngere Großeltern (48 bis 59 Jahre) häufiger gleichzeitig erwerbstätig und betreuen Enkelkinder als ältere Großeltern (60 bis 65 Jahre). Großmütter betreuen nicht nur häufiger ihre Enkelkinder, im Vergleich mit Großvätern kombinieren sie dies auch häufiger mit einer Erwerbstätigkeit. Etwa ein Viertel (25,6 Prozent) der Großmütter und ein Fünftel (20,5 Prozent) der Großväter im Alter bis 65 Jahre sind sowohl in Beruf als auch Familie eingebunden (vgl. Kapitel 15).

Insgesamt zeigt sich also, dass Menschen in der zweiten Lebenshälfte im Jahr 2014 häufiger als in früheren Jahren Erwerbsarbeit mit verschiedenen Sorgetätigkeiten (wie Unterstützung aufgrund von gesundheitlichen Einschränkungen oder Enkelkinderbetreuung) kombinieren. Dies kann zu einer Mehrfachbelastung führen, die sich möglicherweise auch darin widerspiegelt, dass im Jahr 2014 im Vergleich zum Jahr 2008 die unter 65-Jährigen im Durchschnitt eine schlechtere Gesundheit berichten (vgl. Kapitel 8 und 11). Diese Entwicklung sollte genau beobachtet werden, auch im Rahmen von Evaluationen des Familienpflegezeitgesetzes und des Gesetzes zur besseren Vereinbarkeit von Familie, Pflege und Beruf. 


\subsubsection{Gleichstellung von Männern und Frauen: Befunde des DEAS 2014}

Die Befunde des DEAS 2014 zeigen, dass in verschiedenen Lebensbereichen zum Teil deutliche Unterschiede zwischen Männern und Frauen in der zweiten Lebenshälfte bestehen.

In der Altersgruppe der 40- bis 65-Jährigen sind Männer im Jahr 2014 immer noch zu einem deutlich größeren Anteil als Frauen erwerbstätig (Männer: 77,5 Prozent, Frauen: 70,7 Prozent, vgl. Kapitel 3). Das reale Äquivalenzeinkommen ist bei den Männern zwischen 1996 und 2014 ein klein wenig stärker angestiegen als bei den Frauen. Die Einkommensungleichheit zwischen Männern und Frauen hat demnach leicht zugenommen. Zudem sind Frauen in der zweiten Lebenshälfte auch im Jahr 2014 weiterhin häufiger von Einkommensarmut betroffen als Männer (Männer: 10,8 Prozent, Frauen: 13,6 Prozent, vgl. Kapitel 6).

Bei 61,1 Prozent aller Paarhaushalte liegt im Jahr 2014 die überwiegende Zuständigkeit für den Haushalt bei der Frau (vgl. Kapitel 13). Lediglich bei knapp einem Drittel der Paarhaushalte (32,4 Prozent) teilen sich Mann und Frau die Hausarbeit zu gleichen Teilen auf. Daran hat sich seit 2008 nicht wesentlich etwas geändert. Je mehr die Frau im Vergleich zum Mann erwerbstätig ist, desto seltener ist sie allein für die Hausarbeit zuständig. Bei den Paarhaushalten, in denen allein der Mann erwerbstätig ist, liegt bei 77,4 Prozent die überwiegende Zuständigkeit für die Hausarbeit bei der Frau. Wenn sowohl der Mann als auch die Frau erwerbstätig sind, die Frau allerdings in Teilzeit, liegt die Quote immer noch bei 72,5 Prozent. Wenn beide erwerbstätig sind und die Frau dabei in Vollzeit arbeitet, sinkt die Quote auf 48,2 Prozent (vgl. Kapitel 13). Eine Erwerbstätigkeit von Frauen (insbesondere eine Vollzeiterwerbstätigkeit) kann also zu mehr Gleichstellung auch in anderen Lebensbereichen führen.

Frauen sind häufiger als Männer an der Unterstützung und Pflege anderer Menschen beteiligt: Die Unterstützungsquote bei den Frauen im Jahr 2014 beträgt 18,8 Prozent gegenüber 13,3 Prozent bei den Männern. Der Unterschied zwi- schen Männern und Frauen ist hierbei zwischen 1996 und 2014 noch angewachsen (vgl. Kapitel 12). Pflege ist demnach noch immer mit einer ungleichen Arbeitsteilung zwischen Männern und Frauen verbunden.

Die Kontakthäufigkeit zwischen Müttern und ihren erwachsenen Kindern ist größer als bei Vätern: Im Jahr 2014 haben 84,0 Prozent der Mütter und 72,8 Prozent der Väter mindestens wöchentlich Kontakt zu ihren Kindern. Dieser Unterschied hat sich über die Zeit eher vergrößert. Mütter (92,3 Prozent) berichten auch häufiger über eine enge Verbundenheit mit den erwachsenen Kindern als Väter (84,7 Prozent, Kapitel 14). Bezeichnende Unterschiede zwischen Männern und Frauen zeigen sich, sofern es sich um Großeltern handelt, auch in der Einschätzung der Beziehung zu den Enkeln (vgl. Kapitel 15). Erstens ist der Anteil der Großmütter, die die Großelternrolle im Jahr 2014 als ,sehr wichtig' bezeichnen (62,6 Prozent), deutlich größer als der Anteil der Großväter (46,6 Prozent). Zweitens schätzen Großmütter deutlich häufiger als Großväter die Beziehung zu den Enkelkindern als ,eng' oder, sehr eng' ein (74,1 bzw. 62,0 Prozent). Drittens haben Großmütter zu einem größeren Anteil (31,2 Prozent) mindestens wöchentlichen Kontakt zu den Enkeln als Großväter (25,2 Prozent). Dieser Unterschied konnte 2008 noch nicht festgestellt werden. Frauen haben zudem häufiger als Männer einen engen und unterstützenden Freundeskreis (vgl. Kapitel 17). Mit dem Älterwerden kann dies im Hinblick auf die gegenseitige Unterstützung und Hilfe ein entscheidender Faktor dafür sein, länger selbstständig leben zu können.

Frauen berichten im Jahr $2014 \mathrm{zu}$ einem größeren Anteil zumindest leichte depressive Symptome (33,6 Prozent) als Männer (25,9 Prozent). Im hohen Alter ist dieser Unterschied zwischen Frauen und Männern noch größer als über alle Altersgruppen hinweg (vgl. Kapitel 11). Von den Frauen im hohen Alter (70- bis 85-Jährige) gibt ein kleinerer Anteil eine hohe Lebenszufriedenheit an als von den Männern im hohen Alter (Frauen: 76,7 Prozent und Männer: 84,3 Prozent). Bei den Frauen ist der Anteil derjenigen mit guter funktionaler Gesundheit kleiner als bei den Männern (64,2 Prozent bzw. 
73,9 Prozent, vgl. Kapitel 8). Es zeigt sich also, dass es Frauen insbesondere im hohen Alter gesundheitlich und emotional schlechter geht als Männern. Dies könnte auch ein Ausdruck von erlebter Verwitwung sein, von der Frauen wegen ihrer höheren Lebenserwartung häufiger betroffen sind als Männer.

Es gibt auch einige Lebensbereiche, bei denen es keinen oder kaum einen Unterschied zwischen Männern und Frauen gibt. So unterscheiden sich Männer und Frauen nur unwesentlich bei der Einschätzung der eigenen Gesundheit (vgl. Kapitel 10), bei der Lebenszufriedenheit (außer im hohen Alter, vgl. Kapitel 11) und bei der Anzahl der berichteten Erkrankungen (vgl. Kapitel 8). Dennoch zeigen die hier zusammengestellten Befunde, dass die Lebenswirklichkeit in der zweiten Lebenshälfte in Deutschland nach wie vor stark von Unterschieden zwischen Männern und Frauen geprägt ist. Bedenklich ist dabei insbesondere, dass sich die allermeisten der hier berichteten Unterschiede im Zeitverlauf nicht wesentlich verringert haben. Im Großen und Ganzen kann von einer Angleichung der Lebensumstände zwischen Männern und Frauen zumindest bei den hier untersuchten Aspekten keine Rede sein. Eher im Gegenteil: In einzelnen Bereichen haben sich die Unterschiede zwischen Männern und Frauen sogar verstärkt.

\subsubsection{Selbstständiges Wohnen im Alter: Befunde des DEAS 2014}

Im Jahr 2014 lebte nur ein kleiner Teil der älteren Menschen in einer barrierereduzierten Wohnung: Von den 70- bis 85-Jährigen wohnten lediglich 5,6 Prozent in einer solchen Wohnung. Diese niedrige Quote steht in Einklang mit den Befunden verschiedener anderer Studien (für einen Überblick vgl. Prognos 2014). Bedenklich ist, dass selbst von den mobilitätseingeschränkten Menschen (die angeben, eine Gehilfe, einen Rollator, einen Rollstuhl oder Ähnliches zu nutzen), nur 6,9 Prozent in einer barrierereduzierten Wohnung lebten (vgl. Kapitel 19). Zwar muss nicht jeder ältere Mensch in einer barrierereduzierten oder -freien Wohnung leben.
Es ist jedoch bekannt, dass ein nicht unwesentlicher Anteil von Umzügen in eine stationäre Pflegeeinrichtung vermieden oder zumindest hinausgezögert werden könnte, wenn die Wohnung der betroffenen Person barrierereduziert oder -frei gestaltet würde (Bundesinstitut für Bau- Stadt- und Raumforschung 2014).

Die Möglichkeiten der Bundespolitik, auf direktem Wege mehr barrierereduzierte Wohnungen zu schaffen und Einfluss auf die Infrastruktur in den Städten und Gemeinden zu nehmen, sind im deutschen föderalen System begrenzt. Hinsichtlich des barrierereduzierten Wohnens sind Ratgeber und Modellprojekte sicherlich sinnvoll, können aber auch nicht die größte Hürde beseitigen: Für viele Menschen ist ein entsprechender Umbau der Wohnung schlicht zu teuer. Es sollte deshalb verstärkt darüber nachgedacht werden, wie die Kosten eines Wohnungsumbaus verringert und auf mehrere Schultern verteilt werden könnten.

Trotz der geringen Verbreitung barrierereduzierter Wohnungen schätzten 89,7 Prozent der 40- bis 85-Jährigen im Jahr 2014 ihre Wohnsituation insgesamt als gut ein (vgl. Kapitel 19). Auch die Bewertung der Infrastruktur im Wohnumfeld ist gut. Jeweils etwa drei Viertel der Personen gaben im Jahr 2014 an, über ausreichende Einkaufsmöglichkeiten $\mathrm{zu}$ verfügen (76,3 Prozent), genügend Ärzte und Apotheken im Wohnviertel zu haben (80,3 Prozent) und im Wohnviertel eine gute Anbindung an den Nahverkehr zu haben (72,8 Prozent). Von den Personen, die wegen einer körperlichen Einschränkung eine Mobilitätshilfe benutzen, ist der Anteil derjenigen, die die Ausstattung ihres Wohnumfelds mit Einkaufsmöglichkeiten und mit Ärzten und Apotheken als ausreichend erachten, jedoch etwas kleiner (vgl. Kapitel 20). Dies ist ein Hinweis darauf, dass das Wohnumfeld nicht ausreichend altersgerecht gestaltet und ausgestattet ist.

In den letzten Jahren ist die Nachbarschaft verstärkt in den Blick der Politik für ältere Menschen geraten. Im Mix der verschiedenen Hilfe- und Unterstützungsleistungen für ältere Menschen spielt die informelle Nachbarschaftshilfe durchaus eine Rolle (Höpflinger 2012; Tesch-Römer 2010). Die Befunde des DEAS 2014 
belegen eindrucksvoll die große Bedeutung, die die Nachbarschaft für das Wohlbefinden und für die Wohnzufriedenheit hat. Fast die Hälfte der 40 - bis 85 -Jährigen (45,6 Prozent) hatte im Jahr 2014 engen oder sehr engen Kontakt zu den Nachbarinnen und Nachbarn. Allerdings haben Menschen, die in ihrer Mobilität eingeschränkt sind, weniger Personen in der Nachbarschaft, die sie um einen Gefallen bitten könnten, als Personen ohne Einschränkungen (vgl. Kapitel 21).

Die Befunde des DEAS 2014 zeigen, dass Nachbarschaften da besser funktionieren, wo mehr Ressourcen zur Verfügung stehen: zum Beispiel in besseren Wohngegenden und bei gesundheitlich und in ihrer Mobilität weniger eingeschränkten Menschen. In einfachen Wohnlagen haben im Jahr 2014 etwa ein Fünftel (21,4 Prozent) keinen oder nur eine Person in der Nachbarschaft, die für einen Gefallen in Frage käme - in gehobenen Wohnlagen trifft dies nur auf 7,9 Prozent zu. In der Regel können in einem nachbarschaftlichen Hilfe- und Unterstützungsmix Benachteiligungen nicht ausgeglichen werden, denn nachbarschaftliche Unterstützung ist in hohem Maße auf Reziprozität angelegt: Menschen, die dauerhaft weniger geben können als andere, bekommen auch weniger Hilfe als andere - obwohl gerade sie die Hilfe besonders nötig hätten. Das Potenzial, Hilfe und Unterstützung aus der Nachbarschaft zu erhalten, ist also in der Bevölkerung ungleich verteilt.

Politisch kann man daraus folgern, dass es großer Anstrengungen bedarf, nachbarschaftliche Unterstützung auch für sozial Benachteiligte $\mathrm{zu}$ sichern. Inzwischen wird in verschiedenen Modellprojekten der aufsuchenden Gemeinwesenarbeit versucht, zurückgezogen lebende alte Menschen mit Hilfebedarf in Kontakt mit Hilfsangeboten zu bringen (Schubert, Abels, Papenfuß, Spieckermann, \& Veil 2015). Darüber hinaus bestätigen die berichteten DEAS-Ergebnisse 2014, dass für die gegenseitige Hilfe und Unterstützung in Nachbarschaften dasselbe gilt wie für das freiwillige Engagement: Ihre Entstehung ist keine Selbstverständlichkeit, sondern benötigt politische Anstrengungen und Investitionen in förderliche Rahmenbedingungen.

\subsection{Alter nachrangig - Hauptsache, gut gebildet?}

Beim Vergleich der politischen Ziele in der Alternspolitik einerseits und den empirischen Befunden des DEAS 2014 andererseits hat sich bereits angedeutet, dass nicht allein das chronologische Alter die Lebenssituation in der zweiten Lebenshälfte bestimmt, sondern auch eine Reihe anderer Faktoren. Für viele Aspekte des Lebens ist das Lebensalter einer Person sogar weniger relevant als etwa ihre Familiensituation, ihr (vormaliger) Beruf, ihre Einkommensverhältnisse und ihre allgemeine soziale Lage. Einer der wichtigsten Faktoren ist der Bildungsstand, also das Ergebnis des Bildungsverlaufs in Kindheit, Jugend und jungem Erwachsenenalter. Der Bildungsstand prägt die Lebenssituation in vielen Hinsichten stärker als das Lebensalter. Das vorliegende Buch gibt Anhaltspunkte dafür, inwiefern der Bildungsstand und das Alter mit verschieden günstig oder ungünstig ausgeprägten Lebenssituationen einhergehen können.

Erwerbstätigkeit und Einkommen: In Kapitel 3 wird gezeigt, dass der Erwerbsstatus sowohl stark vom Alter als auch vom Bildungsstand abhängt. Im Hinblick auf zeitliche und nervliche Belastungen (Stress) sowie auf körperliche Belastungen bei der Erwerbstätigkeit unterscheiden sich vor allem die 54- bis 59-Jährigen, aber auch die 60- bis 65-Jährigen nur wenig von den Befragten im mittleren Erwachsenenalter. Zwischen den Bildungsgruppen finden sich jedoch deutliche Unterschiede beim Stress und den körperlichen Belastungen im Erwerbsleben. Die objektiven Armutsquoten unterscheiden sich in hohem Maße zwischen verschiedenen Bildungsgruppen und in geringerem Maße zwischen den Altersgruppen. Das gleiche gilt für die subjektiven Armutsquoten (vgl. Kapitel 6). 
Gesundheit: Gesundheitsbezogene Indikatoren hängen in der Regel sowohl vom Bildungsstand als auch vom Alter ab. Dies zeigt sich deutlich bei der Zahl der berichteten Erkrankungen und bei den berichteten gesundheitlichen Einschränkungen: Bei beidem gibt es Alters- und Bildungsunterschiede, dabei sind der Alters- und der Bildungseffekt in etwa gleich groß (vgl. Kapitel 8). Auch bei der Einschätzung des eigenen Gesundheitszustands (subjektive Gesundheit) gibt es Bildungs- und Altersunterschiede - die Altersunterschiede sind bei der subjektiven Gesundheit allerdings etwas weniger ausgeprägt als die Bildungsunterschiede (vgl. Kapitel 10). Dasselbe trifft zu für Lebenszufriedenheit und das Auftreten depressiver Symptome (vgl. Kapitel 11). Ein großer Bildungseffekt zeigt sich bei der körperlichen Aktivität: Der Anteil von Menschen mit hoher Bildung, die mehrmals wöchentlich körperlich aktiv sind, ist mehr als doppelt so groß wie der entsprechende Anteil von Menschen mit niedriger Bildung. Die Unterschiede zwischen den Altersgruppen hingegen sind bei der körperlichen Aktivität geringer als zwischen den Bildungsgruppen (vgl. Kapitel 9).

Ehrenamtliches Engagement: Ein besonders starker Einfluss des Bildungsstandes zeigt sich beim ehrenamtlichen Engagement: Unter den Menschen mit einem hohem Bildungsstand ist der Anteil der Engagierten deutlich höher als unter den Menschen mit einem niedrigen Bildungsstand. Die Unterschiede zwischen den Altersgruppen sind beim ehrenamtlichen Engagement dagegen geringer als zwischen den Bildungsgruppen (vgl. Kapitel 5).

Soziale Integration: Bei der sozialen Integration durch Freundschaften spielen sowohl der Bildungsstand als auch das Alter eine Rolle. Menschen mit einem höheren Bildungsstand geben zu einem deutlich größeren Anteil als Menschen mit einem niedrigeren Bildungsstand an, Freundinnen und Freunde als Partner für Freizeitaktivitäten, im sozialen Netzwerk, als Quelle für Rat und als Quelle für Trost zu haben. Menschen im hohen Alter berichten zu einem deutlich geringeren Anteil von entsprechenden freundschaftlichen Beziehungen als Menschen im mittleren Lebensalter. Bildungsstand und Alter haben dabei ein in etwa ähnliches Gewicht (vgl. Kapitel 17).

Intergenerationale Beziehungen: Auch die Nähe zum nächstwohnenden Kind ist bei Eltern unterschiedlichen Bildungsstands und unterschiedlichen Alters nicht gleichverteilt: Je niedriger der Bildungsstand, desto wahrscheinlicher wohnt eine Person am selben Ort wie das nächstwohnende erwachsene Kind. Und: Je älter eine Person ist, desto wahrscheinlicher wohnt sie am selben Ort wie das nächstwohnende erwachsene Kind. Der Bildungsunterschied ist hier etwas größer als der Altersunterschied (vgl. Kapitel 14). Der Zusammenhang mit dem Bildungsstand ist auch bei der Einschätzung des Verhältnisses von Alt und Jung größer als der Zusammenhang mit dem Alter. Die Befragten sollten jeweils angeben, inwieweit sie den Aussagen „Die Älteren kümmern sich zu wenig um die Zukunft der Jüngeren“ und „Die Jüngeren kümmern sich zu wenig um die Älteren“" zustimmen. Die Zustimmungsquoten unterscheiden sich zwischen den verschiedenen Bildungsgruppen deutlich stärker als zwischen den verschiedenen Altersgruppen (vgl. Kapitel 23).

Altersbilder: Sowohl gewinn- als auch verlustorientierte Altersbilder hängen vom Alter und vom Bildungsstand ab, allerdings unterschiedlich stark (vgl. Kapitel 22). Von den Menschen mit einem hohen Bildungsstand sieht ein deutlich größerer Anteil das Älterwerden als persönliche Weiterentwicklung an als von den Menschen mit einem niedrigen Bildungsstand. Die 70- bis 85-Jährigen sehen das Älterwerden zu einem geringeren Anteil als persönliche Weiterentwicklung an als die 40- bis 54-Jährigen. Der Bildungsunterschied in der Wahrnehmung des Älterwerdens als persönliche Weiterentwicklung ist dabei größer als der Altersunterschied. Bei der Wahrnehmung des Älterwerdens als körperlicher Verlust spielt hingegen das Alter eine etwas größere Rolle als der Bildungsstand. 
Tabelle 25-1: Unterschiede zwischen Altersgruppen und Bildungsgruppen in verschiedenen Bereichen

\begin{tabular}{lll}
\hline $\begin{array}{l}\text { Bereiche, in denen Unterschiede } \\
\text { zwischen Bildungsgruppen etwa } \\
\text { gleich groß sind wie Unterschiede } \\
\text { zwischen Altersgruppen }\end{array}$ & $\begin{array}{l}\text { Bereiche, in denen Unterschiede } \\
\text { zwischen Bildungsgruppen größer } \\
\text { sind als Unterschiede zwischen } \\
\text { Altersgruppen }\end{array}$ & $\begin{array}{l}\text { Bereich, in denen Unterschiede } \\
\text { zwischen Bildungsgruppen kleiner } \\
\text { sind als Unterschiede zwischen } \\
\text { Altersgruppen }\end{array}$ \\
\hline $\begin{array}{l}\text { Erwerbsstatus } \\
\text { Zahl der berichteten Erkrankungen }\end{array}$ & $\begin{array}{l}\text { Stress bei der Erwerbsarbeit } \\
\text { Kërperliche Belastung bei der }\end{array}$ & $\begin{array}{l}\text { Altersbilder: Alter als körperlicher } \\
\text { Verlust }\end{array}$ \\
Soziale Integration durch & Erwerbsarbeit \\
Freundschaften & Objektive Armutsquote \\
& Subjektive Armutsquote \\
& $\begin{array}{l}\text { Einschätzung der eigenen Ge- } \\
\text { sundheit }\end{array}$ \\
& Lebenszufriedenheit \\
& Auftreten depressiver Symptome \\
& Körperliche Aktivität \\
& Ehrenamtliches Engagement \\
& Nähe zum nächstwohnenden \\
erwachsenen Kind & \\
Einschätzung des Verhältnisses \\
von Alt und Jung \\
Altersbilder: Alter als persönliche \\
Weiterentwicklung \\
\end{tabular}

Insgesamt wird deutlich, dass die meisten der Indikatoren, bei denen in diesem Buch die Korrelation mit dem Alter und mit dem Bildungsstand geprüft wurde, sowohl mit dem Alter als auch mit dem Bildungsstand zusammenhängen. Die Anzahl der Variablen, die dabei stärker mit dem Bildungsstand als mit dem Alter korrelieren, ist jedoch deutlich größer als die Anzahl der Variablen, bei denen die Korrelationen in etwa gleich stark sind oder in denen das Alter den größeren Einfluss hat.

Als eine Folge der Bildungsexpansion in den 1970er Jahren haben jüngere Kohorten im DEAS 2014 im Durchschnitt einen höheren Bildungsstand als ältere Kohorten. Bildungstand und Alter sind selbst miteinander konfundiert, ihre Wirkungen auf eine dritte Variable können deshalb nicht ohne weiteres miteinander verglichen werden. In den Analysen sind daher Unterschiede zwischen den Bildungsgruppen für die Altersgruppen kontrolliert berechnet worden (vgl. Kapitel 2) - alle berichteten Bildungsun- terschiede sind also nicht auf die Kohortenzugehörigkeit zurückzuführen. Es zeigt sich, dass der Bildungsstand fast mit allen untersuchten Variablen in starkem Maße korreliert, das Alter hingegen in den meisten Lebensbereichen eine geringere Rolle spielt. Daraus kann gefolgert werden, dass sich innerhalb der Gruppe der 40bis 85-Jährigen ältere und jüngere Menschen in vielerlei Hinsicht weniger stark unterscheiden als gemeinhin vermutet wird. Unterschiede und Ungleichheiten bestehen weniger zwischen Alt und Jung als zwischen Menschen mit unterschiedlichem Bildungsstand.

Die in vielen Bereichen überraschend geringe Bedeutung von Altersunterschieden kann als eine Folge der stetigen Verlängerung der durchschnittlichen Lebenserwartung interpretiert werden. Im Durchschnitt leben die Menschen länger, bleiben länger gesund, sind länger innerhalb oder außerhalb der Familie engagiert und sind materiell besser abgesichert. Es scheint, als ob sich dadurch die mittlere Lebensphase für viele Menschen in man- 
cher Hinsicht bis ins achte und neunte Lebensjahrzehnt hinein verlängert hätte.

Dass sich in den Auswertungen dieses Buches die verschiedenen Altersgruppen in vielen Lebensbereichen relativ wenig voneinander unterscheiden, liegt vermutlich auch daran, dass beim DEAS 2014 keine Personen befragt wurden, die älter als 85 Jahre sind. Aufgrund des Anstiegs der Lebenserwartung in Deutschland und der damit verbundenen Veränderung von Lebensverläufen treten inzwischen bei vielen Menschen in Deutschland entscheidende Veränderungen aufgrund des Alters erst im sehr hohen Alter auf, also eher nach dem 85. Lebensjahr. Menschen, die älter als 85 Jahre alt sind, unterscheiden sich deshalb möglicherweise aufgrund ihres Alters stärker von den jüngeren Altersgruppen als die jüngeren Altersgruppen es untereinander tun.

Bedenklich ist jedoch, wie groß in den meisten Lebensbereichen die Unterschiede zwischen den verschiedenen Bildungsgruppen sind. Der DEAS 2014 bestätigt damit eindrucksvoll die Erkenntnis, dass der einmal erworbene Bildungsstand den weiteren Lebensverlauf in hohem Maße prägt. Für die Politik lässt sich daraus einmal mehr der Schluss ziehen, dass das Augenmerk weiterhin stark auf den Abbau sozialer Ungleichheiten gerichtet sein sollte.

\subsection{Fazit}

Die oben stehenden Ausführungen machen deutlich, dass es sinnvoll ist, Befunde aus verschiedenen Kapiteln dieses Buches zusammenzustellen, miteinander in Beziehung zu setzen und auf diese Weise themenbezogene Gesamtbilder zu erstellen. Dabei wird offensichtlich, dass die Themen der Alternspolitik Querschnittsthemen sind und Berührungspunkte zu mehreren Politikfeldern haben.

Die Befunde des DEAS 2014 zeigen, dass sich die lange gültige Dreiteilung des Lebenslaufs auflöst: In vielerlei Hinsicht kann von einer Ausweitung der mittleren Lebensphase gesprochen werden. Angesichts der Verlängerung der Lebenserwartung ist diese Entwicklung auch nicht überraschend. Der Übergang in den Ruhestand, der für die meisten Erwerbstätigen im siebten Lebensjahrzehnt stattfindet, verändert in unserer erwerbsarbeitszentrierten Gesellschaft zwar Einiges im Alltag der Menschen, aber eben auch nicht alles: In vielen Bereichen gibt es über die Altersgruppen hinweg bis ins neunte Lebensjahrzehnt hinein viel Kontinuität.

Allerdings vollzieht sich diese Ausweitung der mittleren Lebensphase nicht für alle Menschen in gleicher Weise. In der im DEAS 2014 untersuchten Altersspanne von 40 bis 85 Jahren ist ja gerade nicht das Lebensalter der bedeutendste Unterscheidungsfaktor zwischen sozialen Gruppen - sehr viel bedeutsamer ist der Bildungsstand. Dies bedeutet zweierlei: Zum einen lohnen sich die Aufwendungen für Bildung in frühen Lebensabschnitten, sie wirken über den gesamten Lebenslauf bis in die Phase des Alters. Andererseits sollten gerade Menschen, die in Kindheit und Jugend nur einen geringen Bildungsabschluss erreichen konnten, im Verlauf des Älterwerdens Unterstützung erhalten, um ein gutes Altwerden in sozialer Integration und mit gesellschaftlicher Teilhabe zu erleben. 


\section{Literatur}

Bäcker, G. (2015). Jahresrückblick Alterssicherungspolitik. Zeitschrift für Sozialreform, 61(1), 4-13.

Berner, F., \& Hagen, C. (2015). Herausforderungen einer lokalen Politik für ältere und mit älteren Menschen. Pro Alter, 47(6), 19-21.

Bundesinstitut für Bau-, Stadt- und Raumforschung. (2014). Potenzialanalyse altersgerechter Wohnungsanpassung. Bonn: Bundesinstitut für Bau-, Stadt- und Raumforschung.

Bundesministerium für Arbeit und Soziales (2014). Zweiter Bericht der Bundesregierung gemä $\beta$ \& 154 Absatz 4 Sechstes Buch Sozialgesetzbuch zur Anhebung der Regelaltersgrenze auf 67 Jahre. Berlin: Bundesministerium für Arbeit und Soziales.

Czepek, J., \& Weber, E. (2015). Flexibilität beim Übergang in die Rente. Aktuelle Berichte des Instituts für Arbeitsmarkt- und Berufsforschung Nürnberg: Institut für Arbeitsmarkt- und Berufsforschung.

Engstler, H., \& Romeu Gordo, L. (2014). Arbeiten im Ruhestand. Entwicklung, Faktoren und Motive der Erwerbstätigkeit von Altersrentenbeziehern. In: E. Kistler \& F. Trischler (Hrsg.) Reformen auf dem Arbeitsmarkt und in der Alterssicherung - Folgen für die Einkunftslage im Alter (S. 115-147). Düsseldorf: Hans-BöcklerStiftung.

Höpflinger, F. (2012). Zuhause lebende Menschen im Alter - soziale Netzwerke, Gesundheit und ambulan- te Unterstützung. In: P. Perrig-Chiello \& F. Höpflinger (Hrsg.) Pflegende Angehörige älterer Menschen (S. 63107). Bern: Huber.

Prognos (2014). Evaluation des KfW-Programms „Altersgerecht umbauen". Basel.

Rothgang, H., Müller, R., \& Unger, R. (2012). Themenreport „Pflege 2030“. Gütersloh: Bertelsmann Stiftung.

Scherger, S., \& Hokema, A. (2014). Arbeiten müssen, können oder wollen? Erwerbstätigkeit jenseits der Rentengrenze in Deutschland. In: K. Kaudelka \& G. Insenbort (Hrsg.) Altern ist Zukunft! Leben und arbeiten in einer alternden Gesellschaft (S. 143-157). Bielefeld: transcript.

Schubert, H., Abels, S., Papenfuß, K., Spieckermann, H., $\&$ Veil, K. (2015). Neuer Infrastrukturansatz für die sozialräumliche Altenhilfe. In: A. van Rießen, C. Bleck \& R. Knopp (Hrsg.) Sozialer Raum und Alter(n): Zugänge, Verläufe und Übergänge sozialräumlicher Handlungsforschung (S. 131-156). Wiesbaden: Springer VS.

Statistisches Bundesamt (2013). Pflegestatistik 2013, Deutschlandergebnisse. Wiesbaden: Statistisches Bundesamt.

Tesch-Römer, C. (2010). Soziale Beziehungen alter Menschen. Stuttgart: Kohlhammer.

Wetzstein, M., Rommel, A., \& Lange, C. (2015). Pflegende Angehörige - Deutschlands größter Pflegedienst. GBE Kompakt, 6(3).

Open Access Dieses Kapitel wird unter der Creative Commons Namensnennung 2.5 International Lizenz (http://creativecommons.org/licenses/by/2.5/deed.de) veröffentlicht, welche die Nutzung, Vervielfältigung, Bearbeitung, Verbreitung und Wiedergabe in jeglichem Medium und Format erlaubt, sofern Sie den/die ursprünglichen Autor(en) und die Quelle ordnungsgemäß nennen, einen Link zur Creative Commons Lizenz beifügen und angeben, ob Änderungen vorgenommen wurden.

Die in diesem Kapitel enthaltenen Bilder und sonstiges Drittmaterial unterliegen ebenfalls der genannten Creative Commons Lizenz, sofern sich aus der Abbildungslegende nichts anderes ergibt. Sofern das betreffende Material nicht unter der genannten Creative Commons Lizenz steht und die betreffende Handlung nicht nach gesetzlichen Vorschriften erlaubt ist, ist für die oben aufgeführten Weiterverwendungen des Materials die Einwilligung des jeweiligen Rechteinhabers einzuholen. 